\title{
Time to Re-Think Broca: Extent of Resection and Neurological Outcome in Patients Harboring Tumors in the Dominant Inferior Frontal Gyrus
}

Paola Suarez-Meade ( $\sim$ meade.paola@mayo.edu )

Mayo Clinic's Campus in Florida https://orcid.org/0000-0002-3638-6799

Lina Marenco-Hillembrand

Mayo Clinic's Campus in Florida

David Sabsevitz

Mayo Clinic's Campus in Florida

Lela Okromelidze

Mayo Clinic's Campus in Florida

Blake Perkidis

Duke University

Wendy J. Sherman

Mayo Clinic's Campus in Florida

Alfredo Quinones-Hinojosa

Mayo Clinic's Campus in Florida

Erik H. Middlebrooks

Mayo Clinic's Campus in Florida

Kaisorn L. Chaichana

Mayo Clinic's Campus in Florida

\section{Research Article}

Keywords: "Broca's area", neuropsychological, glioma, morbidity

Posted Date: August 12th, 2021

DOl: https://doi.org/10.21203/rs.3.rs-788969/v1

License: (c) (i) This work is licensed under a Creative Commons Attribution 4.0 International License.

Read Full License 


\section{Abstract}

Introduction/Purpose: There is a general lack of consensus onboth the anatomic definition and function of Broca's area. Given the belief that this region plays a critical role in expressive language, resective surgery is often avoided topreserve function. However, the putative role of Broca's area in speech production has been recently challenged.The current study aims to investigatethe feasibilityof glioma resection and neurological outcomes in "Broca's area" in 15 patients.

Methods: We report a feasibility study describing the resection of gliomas within the IFG. Awake brain surgery for resection with mapping and intraoperative neuropsychological evaluation was carried out in all individuals.

Results: All included patientshad tumors located in traditional "Broca's area" and eight patients (53.33\%) had tumors that additionally extendedinto the insula and subinsular regions. During stimulation, positive speech-language sites within the IFG were identified in ten patients. Two patients (13.33\%) experienced a declinein naming during intraoperative cognitive monitoring and thirteen $(86.66 \%)$ had a stable performance throughout surgery. With all patients had recovery of language functions at a two-week follow up. Extent of resection was stratified in anatomical regions within the IFG, being the pOr the area with the greatest EOR (97.4\%), followed by the pT (84.1\%), pOp (83.8\%), and vPMC (80\%).

Conclusion: The belief that Broca's area is not safe to resect is challenged. Adequate mapping and careful patient selection allow maximum safe resection of tumors located in thetraditional "Broca's area",with low risk of postoperative morbidity.

\section{Introduction}

The understanding of the neural basis of speech-language has evolved and expanded since the traditional models were described. The classical anatomical model of language was first represented in the works of Dr. Broca [1]. He describes a localizationist theory, where discrete cortical areas of the brain are responsible for speech production, traditionally referencing "Broca's area"; located on the pars opercularis (pOp) and pars triangularis (pT) (Brodmann's areas 44 and 45) of the frontal operculum within the dominant inferior frontal gyrus (IFG) (Table 1.). Historically, Broca's area was associated with speech motor functions and, when damaged, resulted in non-fluent aphasia characterized by severely reduced, effortful speech, and word-finding difficulty. However, there is accumulating evidence that challenges the notion that Broca's area is critical for speech production [2-4,6]. We demonstrate that mapping the IFG has important interindividual variability and that corticectomy and resection of areas that are thought to be essential for speech is possible.

Lesions restricted to the traditional cortical Broca's area, often result in mild and more transient non-fluent aphasia, whereas more severe and permanent speech deficits require lesions to extend posteriorly to the vPMC and deeper to the underlying white matter tracts. The theory of a subcortical "bottleneck" of white matter tracts deep to the cortical Broca's area, posits that the full spectrum of severe speech deficits from 
damage to IFG is likely, in large part, due to damage to multiple eloquent tracts that converge just deep to the Broca area [5]. In fact, a recent re-examination of Broca's original patients using high-resolution MRI revealed extensive subcortical damage that was not originally appreciated by Dr. Broca [3].

Because of the important language eloquence in the IFG patients with tumors in or adjacent to Broca's area were thought to be at higher risk of postoperative language morbidity resulting in the traditional mantra that tumors in this area are often deemed unresectable. In line with the contemporary connectomal description of language networks and the dynamic reorganization of language circuits during disease, we aimed to conduct a feasibility study on glioma surgery within the pOp and pT.

\section{Methods}

\section{Study Design, Setting, and Population}

This study is a single-surgeon consecutive case series, where adult patients harboring tumors within the left IFG who underwent awake surgical resection with mapping between July 2018 and August 2020. Tumor location in Broca's area within the IFG was determined using MR imaging. Broca's area was anatomically defined to be located at the $\mathrm{pT}$ and the pOp, as originally described. Patients were excluded if histopathologic diagnosis was other than glioma, had multifocal disease, were left-handed or had absolute contraindications for awake surgery [7]. Preoperative Karnofsky Performance Status (KPS) and neuropsychological baseline language status were established the day prior to the procedure [8]. Demographic, histologic, imaging, surgical, neuropsychological, and clinical outcomes were included for analysis.

\section{Neuropsychological Evaluation and Intraoperative Functional Language Tasks}

All patients underwent baseline language testing using a tablet-based testing platform for intraoperative mapping (NeuroMapper). Administered subsets were individualized to tumor location and the affected white matter tracts, with picture naming, nonword repetition, and digit repetition being the most utilized tests. Accuracy and reaction time data were collected. Items that were answered correctly within a prespecified time to allow for DCS stimulation mapping were selected for intraoperative evaluation (e.g., < 5-7 seconds). Paradigms were administered during stimulation and continuously throughout active resection of the tumors to monitor cognition. Patient performance was considered as stable when error rate was $<5 \%$ in picture naming responses and cognitive decline was defined as $>5 \%$ error rate compared to baseline.

\section{Surgical Procedure and Intraoperative Mapping}

All patients underwent awake surgery with brain mapping for tumor resection $[9,10]$. Main surgical goals were to achieve maximal safe resection (MSR) and to obtain tissue for diagnosis. Scalp nerve block was done to ensure analgesia before positioning. After dural opening, direct cortical stimulation (DCS) was carried out, starting at $3 \mathrm{~mA}$, increased at $1 \mathrm{~mA}$ intervals, until after discharges were present, positive 
threshold response was established, or reached $9 \mathrm{~mA}$ with negative mapping with the Ojemann stimulator. All sites were stimulated at least three times. After identifying the most convenient surgical corridor, direct subcortical stimulation (DSS) was done. During DCS and DSS, patients performed a picture naming paradigm where they were required to say "This is a" followed by naming the presented picture. The onset of electrical stimulation was timed to just prior to the presentation of the picture and it was continued until a response was made or until 5-7 seconds with no response. The neuropsychological paradigms were administered continuously and throughout the entire active resection. (Table 3.). Tumor location, positive mapping areas, and patient performance throughout surgery determined the extent of resection (EOR) of the lesions. FLAIR was chased on tumors with high-grade characteristics in preoperative MRI. After achieving maximal safe resection (MSR), hemostasis was secured, and the surgical approach was closed in anatomical layers. Postoperative MRI imaging within the first 24 hours after the procedure.

\section{MR Imaging and Extent of Resection Measurements:}

Standard preoperative and immediate postoperative imaging were acquired with and without gadoliniumbased contrast. The preoperative and postoperative post-contrast T1-weighted, T2-weighted, and postcontrast FLAIR images were co-registered using a linear affine registration implemented in Statistical Parametric Mapping (SPM) v12 (https://www.fil.ion.ucl.ac.uk/spm/). Masks were created for enhancing tumor (ET) from the post-contrast T1-weighted images, non-enhancing tumor (NET) based on T2weighted images, and peritumoral edema (PE) based on FLAIR images using ITK-Snap [11]. Using the T1-weighted imaging as reference, normalization to the Montreal Neurological Institute (MNI) template space was performed to generate a transformation warp that was applied to the coregistered T2weighted, FLAIR images, and segmentation masks.

Total and total residual tumor maps were generated based on the sum of the preoperative and postoperative ET and NET maps, respectively. Individual tumor volume was calculated for each mask. Resection was categorized as follows: gross total resection (GTR): resection above $95 \%$; subtotal resection (STR): between 60-95\% tumor resected; partial resection (PR): less than $60 \%$ resection [12]. Individual total and total residual tumor maps were combined into group-level, preoperative and postoperative tumor masks with an integer value for each voxel representing the number of subjects with tumor in that voxel. Cortical masks from Harvard-Oxford Cortical Atlas were used for preoperative and postoperative tumor volume calculations in the vPMC, pOp, pT, and pOr [13-15].Difference between preoperative and postoperative tumor volume difference was calculated for the evaluation of the extent of tumor resection.

Next, to evaluate the relationship of tumor to critical subcortical white matter structures, a tract atlas was generated in MNI space. Tractography was based on a group-averaged dataset from 1021 subjects in the Human Connectome Project (https://www.humanconnectome.org) which was reconstructed with a qspace diffeomorphic reconstruction [16,17].Individual tracts were then generated in DSI Studio (http://dsistudio.labsolver.org). Full acquisition details are available at the Human Connectome Project website. 
Briefly, a multishell diffusion scheme was with b-values of 1000, 2000, and $3000 \mathrm{~s} / \mathrm{mm} 2$ with 90 diffusion directions per shell. Images were acquired with an isotropic resolution of $1.25 \mathrm{~mm}$. A deterministic fiber tracking algorithm was used [18].Tract data was semi-automated based on prior tractography atlas [19].Topology-informed pruning was applied to the tractography with 3 iterations to remove false connections. The residual tumor group map (thresholded at $>13 \%$ or $n>2$ ) was compared to the tract anatomy in DSI Studio [20].

\section{Postoperative Care}

Patients were evaluated two weeks after surgery and at follow-up visit at 3 months. Outcome measures of postoperative KPS, EOR, presence of postoperative language deficits, surgical complications, length of hospital stay were collected. Length of hospital stay was from the time of surgery to the day of discharge.

\section{Compliance with Ethical Standards:}

All procedures performed in this study involving human participants were in accordance with the ethical standards of the institution and the national research committee. This study was carried out under the IRB number: PR16-009946-01.Informed consent was obtained from all individual participants included in the study.

\section{Statistical Analysis}

Descriptive statistics were utilized to detail patient characteristics and postoperative outcomes. Data was expressed as a mean with range for continuous variables and counts with percentages for categorical variables. Student $T$-test was utilized for continuous data. $P<0.05$ was considered statistically significant. All analyses were performed using GraphPad Prism 9.0.2 for Windows (Graphpad Software, La Jolla, California, USA).

\section{Results}

\section{Patient Demographics and Presentation}

A total of 15 consecutive right-handed patients with tumors in the left IFG were included for analysis (Table 2). Lesion location within the IFG was most commonly in the pOp (80\%), followed by the vPMC (53.33\%), pT (33.33\%), and pOr (6.66\%). One lesion extended into the temporal lobe and eight extended into the insula. Fourteen patients (93.33\%) showed some type of speech deficit at diagnosis as determined by review of presenting clinical history or neurologic examination. Patients most often presented with non-fluent aphasia $(n=5,55.55 \%)$, verbal working memory deficits $(n=8,53.33 \%)$, and speech arrest $(n=4,26.66 \%)$.

\section{Surgical Characteristics}


All patients underwent awake brain surgery with tailored pterional craniotomy and negative mapping technique $(n=15)$ (Figure 1.). One patient presented with intraoperative seizures due to DCS that were controlled with cold saline. No other complications were noted. A transcortical corridor was used for access in $13(86.66 \%)$ patients, to minimize exposure of the lenticulostriate arteries. Transcortical corridors were done through areas that showed no language disruption with DCS. Specifically, surgical entry was made through the pOp $(n=7,46.66 \%), p T(n=1,6.66 \%), \operatorname{pOr}(n=1,6.66 \%)$, pOr + PT $(n=1,6.66 \%)$, MFG $(n=2,13.33 \%)$, and STG $(n=1,6.66 \%)$ (Table 3$)$.

\section{Intraoperative Language Mapping}

Patients were evaluated by neuropsychology before and during surgery. Five patients (33.33\%) had no identifiable frontal lobe language sites during DCS while ten (66.66\%) presented errors in picture naming during DCS. Disruption of speech language functions during DCS was commonly found in the vPMC $(n=9,60 \%)$, followed by the pT $(n=3,20 \%)$, and the pOp $(n=3,20 \%)$ (Table 3). For direct subcortical stimulation (DSS), only the SLF $(n=2)$, the IFoF $(n=1)$, and the AF $(n=1)$ tracts resulted in positive findings for speech arrest and phonetic paraphasias (Table No. 3). During continuous neuropsychological monitoring, twelve patients $(86.66 \%)$ remained stable throughout the procedure. Only two patients $(13.33 \%)$ presented with mild decline in naming during surgery, as they presented $>5 \%$ in error rate compared to baseline. Neuropsychology evaluation was useful to identify the limits of the surgery, as resection was stopped in patients that presented with any kind of deficits during active resection to preserve function.

\section{Extent of Resection}

Prior to surgery, median tumor volume was $\left(79.42 \pm 80.14 \mathrm{~cm}^{3}\right)$, while mean residual tumor volume was $\left(39.83 \pm 55.24 \mathrm{~cm}^{3}\right)$ (Figure 2.). Overall, the mean EOR was $60.35 \%( \pm 29.60 \%)$. EOR was stratified in anatomical regions within the IFG, being the pOr the area with the greatest EOR (97.4\%), followed by the pT $(84.1 \%)$, the pOp (83.8\%), and then the VPMC (80\%). Although not statistically significant, EOR for high-grade gliomas (WHO grade III and IV) $(72.93 \pm 17.94)$ was greater when compared to low-grade gliomas (WHO grade I and II) $(60.75 \% \pm 30.51)(p=0.4010)$. Resection was limited by positive mapping areas during DCS, cognitive decline, and involvement of high-risk structures. Residual tumor was commonly located within the insular and subinsular regions as well as the deep margins within critical white matter regions of the centrum semiovale (Figure 3. and Figure 4.).

\section{Postoperative Outcome and Neurological Morbidity}

Outcome data is summarized in Table 4. Patients were hospitalized for $2.33( \pm 1.19)$ days on average. Although recovery was noted in all patients at two-weeks postop compared to baseline, seven patients $(46.66 \%)$ still presented with mild speech and language deficits that were related to tumor growth and not to surgical procedure (Table 3. and Table 4.). Mean postoperative functional status (KPS: $88.66 \pm 8.84$ ) calculated at two weeks after surgery remained the same as the mean preoperative functional status (KPS:88 \pm 8.32). (Table 4.). 


\section{Discussion}

Surgery within "Broca's area" for tumor resection is feasible with very minimal and acceptable postoperative morbidity. In our case series, EOR ranged between $80-97.4 \%$ in tumors located in the IFG. Resection was mainly limited by positive mapping, white matter tract involvement, and insular extension of lesions. Resection in these areas was avoided to prevent injury to vascular and functional structures, which prevented deflection of the results seen after resection in "Broca's area". Moreover, mapping the IFG has important interindividual variability and that corticectomy and resection of areas that are thought to be essential for speech is possible. When compared to presentation at diagnosis, all patients had remarkable recovery of language functions two weeks after surgery and no new surgical-related deficits were noted. These results demonstrate that resection of gliomas in what is described as "Broca's area" is feasible in a carefully selected subset of patients.

For more than a century, the classical model of language organization proposed by Doctors Broca and Wernicke was universally accepted despite many observations that were not accounted for in this incomplete model $[1,21]$. Broca's descriptions led to a localizationist model which placed the speech output epicenter within the posteromedial portion of the IFG (pT, pOp; Brodmann areas 44 and 45) of the dominant hemisphere $[22,23]$. However, Broca's model was largely based upon lesionology and their proponents failed to account that Leborgne's and Lelonge's brains - the so-called "Broca's Brains" showed damage beyond the cortical limits of Broca's area, extending to the insula and to the perisylvian white matter [3]. These overly simplified models attributed fixed functions to specific areas of the brain leading to the general belief that surgical intervention in these "eloquent" areas was impossible [24]. The current understanding of language has advanced towards a connectomal framework which largely recognizes interindividual anatomo-functional variability [25,26]. Current models acknowledge the dynamic and complex subcortical circuitry that interconnect, reshape, and distribute information within the brain $[21,27,28]$.

Preserving function is one of the most important principles in brain tumor surgery, as postoperative morbidity has been demonstrated to impact patient prognosis $[29,30]$.For that reason, surgical resection of tumors located in the dominant IFG and adjacent subcortical areas was traditionally considered implausible. Nonetheless, several authors have proved that resection of Broca's area does not always lead to language deficits [31-36]. Benzgamout et al. reported a series of 7 patients with LGG who underwent awake craniotomy for tumor resection with intraoperative mapping. Speech motor epicenters were found within the ventral and dorsal premotor cortices, the orbitofrontal cortex, and the insula [32]. Similar findings were described by Lubrano et al. who reported sixteen consecutive patients with lesions within Broca's area [33].In this series, they correlated the morphology of the lesion to positive mapping during stimulation of the $\mathrm{pOp}$ and $\mathrm{pT}$, if the lesion was well-circumscribed, positive speech areas within Broca's area were found at a rate of $100 \%$, whilst infiltrative lesions presented positive mapping in Broca's area in only $25 \%$ of cases. In the largest series performed to date, Rolston et al. retrospectively evaluated a series of 43 patients with opercular lesions, 33 of which underwent awake craniotomy with language mapping. After follow-up authors concluded that tumors located within the pOp did not possess any risk 
for developing postoperative language deficits and identified a number of risk factors associated with postoperative deficit appearance [34]. These included presence of preoperative seizures, preoperative language deficits, and decreased extent of resection.

The insula has also been identified as an important center of coordination of the articulation regions. Studies indicate that the dominant anterior insula is likely a critical node in speech output, receiving final word selection from the frontal operculum, conveying it to the cerebellum and basal ganglia and relaying this information to the vPMC and vSMC $[23,38]$. Neuroimaging studies and lesion to this structure indicate its key role in articulatory control and higher-order cognitive aspects of speech [37].In our work, postoperative imaging revealed that most residual tumors were located within the insula $(n=9,60 \%)$ and in the vPMC $(n=6,40 \%)$. Resection of the insular component of the tumors was avoided due to eloquent tissue and to critical microvasculature for language and motor systems that pose a high-risk of stroke during surgery.

The VPMC has been repeatedly identified as the area with the highest rate of speech arrest upon stimulation, occurring in $83 \%$ of cases after stimulation of the vPMC in the dominant hemisphere. In 2014 , Tate et al. challenged the traditional theories of language organization after performing a probabilistic map of critical functional regions within the human cerebral cortex of 165 patients with LGG, including Broca's area. Interestingly, speech arrest was localized within the vPMC, not Broca's [4]. Their results correlated with other studies where speech deficits were localized in the vPMC $[6,35,38,39]$. Although the role of the left vPMC in language is poorly understood it may have a role in the control of the initiation of complex movements, allowing speech articulation including coordination of the tongue, lips and pharynx. The limited plastic potential of the left vPMC as well as the critical underlying white matter tracts, makes it a high-risk area for surgical resection [40]. Thus, we conclude that stimulation of the VPMC causes higher rate of speech arrest than Broca's; which challenges the prevailing notion of Broca's area as the quintessential motor speech epicenter.

The results presented herein differ from the classical localizationist model and support the use of the contemporary hodotopical basis of language for tumor surgery. Given the lack of anatomic specificity, and its historically oversimplified functional role,the field may benefit by no longer using the moniker of "Broca's area". More specific anatomic and functional anatomy of the IFG cortex, as well as differentiation from underlying white matter tracts, may add to clarity and reproducibility of surgical literature.

\section{Strengths and Limitations.}

While the results of our study provide insightful information on the safety profile of surgical resections in the IFG, it also carries some limitations. The retrospective nature of the study, which limited the author's capacity to homogenize available data, small sample size, and heterogeneous pathology presented in our cohort have to be considered. This study also carries various strengths. Surgical data was obtained from a single surgeon, reducing variability and bias in our results. Real-time monitoring of speech and language was used as a primary outcome variable, increasing the sensitivity of detecting surgical 
induced injury rather than subacute surgical effects (e.g., edema). Neuropsychological testing was carried out a novel tablet-based platform that is OR-friendly and allows individualized testing in a specific and safe manner.

\section{Concluding Remarks:}

Awake resection of tumors located within dominant inferior frontal gyrus can be safely performed with extremely low complication rates and minimal postoperative morbidity. In the presence of tumors, surgery is limited by identification of functional areas and not by the anatomical limits of what is thought to be "Broca's area".

\section{Declarations}

Data availability statement: The data that support the findings of this study are available from the corresponding author, $\mathrm{KLC}$, upon reasonable request.

Conflict of interest: Authors declare no conflict of interest

\section{Author contribution:}

Conceptualization and Study Design: PSM, LMH, DS, EHM, KLC

Data Acquisition: PSM, LMH, DS, LO, BP, EHM, KLC

Data Interpretation: PSM, LMH, DS, EHM, WJS, AQH, KLC

Writing: PSM, LMH, DS, EHM

Review and Editing: DS, WJS, AQH, EHM, KLC

\section{References}

1. Broca P. Nouvelle Observation d'aphemie produite par une lesion de la moitie posterirure des deuxieme et troisieme circonvolutions frontale. Bull Soc Anat. 1861. (6): 337-393.

2. Kim JH, Amankulor NM, Peck KK, Brennan N, Gutin PH, Holodny Al. Resection of glioma in an fMRIdefined "split" Broca's Area. Neurocase. 2014; 20 (5): 481-486.

3. Dronkers NF, Plaisant O, Iba Zizen MT, Cabanis EA. Paul Broca's Historic Cases: High resolution MR imaging of the brains of Leborgne and Lelong. Brain. 2007; 130 (5): 1432-1441.

4. Tate MC, Herbert G, Moritz-Gasser S, Tate JE, Duffau H. Propabilistic Map of Critical Functional Regions of the Human Cerebral Cortex: Broca's Area Revisited. Brain. 2014; 137 (10): 2773-2782.

5. Griffis JC, Nenert R, Allendorfer JB, Szaflarski JP. Damage to White Matter Bottlenecks Contributes to Language Impairments after Left Hemispheric Stroke. Neuroimag: Clinical. 2017; (14): 552-565 
6. Ferpozzi V, Fornia L, Montagna M, Siodambro C, Castellano A, Borroni P, et al. Broca's Area as a PreArticulatory Phonetic Encoder: Gating the Motor Program. Front Hum Neurosci; 2018: 12:64

7. Hervey-Jumper SL, Li J, Lau D, Molinaro AM, Perry DW, Meng L, et al. Awake Craniotomy to Maximize Glioma Resection: Methods and Technical Nuances Over a 27-year Period. J Neurosurg. 2015; 123 (2): 325-39.

8. Yates JW, Chalmer B, McKegney FP. Evaluation of Patients with Advanced Cancer using the Karnofsky Performance Status. Cancer. 1980; 45 (8): 2220-2224.

9. Eseonu Cl, Rincon-Torroella J, Lee YM, ReFaey K, Tripathi P, Quinones-Hinojosa, A. Intraoperative Seizures in Awake Craniotomy for Perirolandic Glioma Resections that Undergo Cortical Mapping. $J$ Neurol Sug A Cent Eur Neurosurg. 2018. 79 (3): 238-246.

10. ReFaey K, Tripathi S, Bhargav AG, Grewal SS, Middlebrooks EH, Sabsevitz DS, et al. Potential Differences Between Monolingual and Bilingual Patients in Approach and Outcome After Awake Brain Surgery. J Neurooncol. 2020; 148 (3): 587-598.

11. Yushjevich PA, Piven J, Cody Hazlett H, Gimpel Smith R, Ho S, Gee JC, et al. User-guided 3D active contour segmentation of anatomical structures: significantly improved efficiency and reliability. Neuroimage. 2006; 31 (3): 116-28.

12. Chaichana KL, Jusue-Torres I, Navarro-Ramirez R, Raza SM, Pascual-Gallego M, Ibrahim A, et al. Establishing percent resection and residual volume thresholds affecting survival and recurrence for patients with newly diagnosed intracranial glioblastoma. Neuro-Oncology. 2014; 16 (1): $113-22$.

13. Makris N, Goldstein JM, Kennedy D, Hodge Sm, Caviness VS, Faraone SV, et al. Decreased volume of left and total anterior insular lobule in schizophrenia. Schizophr Res. 2006.83 (2-3): 155-71.

14. Frazier JA, Chiu S, Breeze JL, Makris N, Lange N, Kenney DN, et al. Structural brain magnetic resonance imaging of limbic and thalamic volumes in pediatric bipolar disorder. Am J Psychiatry. 2005; 162 (7): 1256-65.

15. Desikan RS, Segonne F, Fischl B, Quinn BT, Dickerson BC, Blacker D, et al. An automated labeling system for subdividing the human cerebral cortex on MRI scans into gyral based regions of interest. Neuroimage. 2006. 31 (2): 968-80.

16. Yeh FC, Tseng WY. NTU-90: a high angular resolution brain atlas constructed by q-space diffeomorphic reconstruction. Neuroimage. 2011; 58L 91-99.

17. 39. Yeh FC, Wedeen VJ, Tseng WY. Generalized q-sampling imaging. IEEE Trans Med Imaging. 2010; 29 (9): 1626-35.

18. Yeh FC, Verstynen TD, Wang Y, Fernandez-Miranda JC, Tseng WY. Deterministic diffusion fiber tracking improved by quantitative anisotropy. PLoS ONE. 2013; 8 (11): 80713

19. Yeh FC, Panesar S, Fernandes D, Meola A, Yoshino M, Fernandez-Miranda JC, et al. Population averaged atlas of the macroscale human structural connectome and its network topology. Neuroimage. 2018; 178: 57-68.

20. Yeh FC, Panesar S, Barrios J, Fernandes D, Abhinav K, Meola A, et al. Automatic removal of false connections in diffusion MRI tractography using topology-informed prining (TIP). Neurotherapeutics. 
2019; $16(1): 52-58$.

21. Duffau H. The Error of Broca: From the Traditional Localizationist Concept to a Connectomal Anatomy of the Human Brain. J Chem Neuroanat. 2018; 89: 73-81.

22. Vanacor CN, Isolan GR, Yu YH, et al. Microsurgical Anatomy of Language. Clin Anat. $2021 ; 34$ (1): 154-168.

23. Stinnet TJ, Reddy V, Zabel MK. Neuroanatomy, Broca. In: StatPearls. Tresure Island (FL): Statpearls Publishing

24. Sawaya R, Hammoud M, Schoppa D, Hess KR, Wu SZ, Shi WM, et al. Neurosurgical Outcomes in a Modern Series of 400 Craniotomies for Treatment of Parenchymal Tumors. Neurosurgery. $1998 ; 42$ (5): 1044-1055.

25. Middlebrooks EH, Yargmurlu K, Szaflarski JP, Rahman M, Bozjurt B. A contemporary framework of language processing in the human brain in the context of preoperative and intraoperative language mapping. Neuroradiology. 2017; 59 (1): 69-87.

26. Yagmurlu K, Middlebrooks EH, Tanriover N, Rhoton AL Jr. Fiber tracts of the dorsal language stream in the human brain. J Neurosurg; 2016: 124 (5): 1396-405.

27. Flinker A, Korzeniewska A, Shestyuk AY, Franaszczuk PJ, Dronkers NF, Knight RT, et al. Redefining the Role of Broca's Area in Speech. PNAS. 2015; 112 (9): 2871-2875.

28. Chang EF, Raygor KP, Berger MS. Contemporary Model of Language Organization: An Overview for Neurosurgeons. Journal of Neurosurgery. 2015; 122: 250-261.

29. Rahman M, Abbatematteo J, De Leo EK, Kubulis PS, Vaziri S, Bova F, et al. The effects of new or worsened postoperative neurological deficits on survival of patients with glioblastoma. J Neurosurg. 2016; 127 (1): 123-131.

30. McGirt MJ, Mukherjee D, Chaichana KL, Than KD, Weingart JD, Quinones-Hinojosa, A. Association of Surgically Acquired Motor and language deficits on overall survival after resection of glioblastoma multiforme. Neurosurgery. 2009; 65 (3): 463-9.

31. Chaichana KL, Jusue-Torres I, Navarro-Ramirez R, Raza SM, Pascual-Gallego M, Ibrahim A, et al. Establishing Percent Resection and Residual Volume Thresholds Affecting Survival and Recurrence for Patients with Newly Diagnosed Intracranial Glioblastoma. Neuro Oncol. 2014; 16 (1): 113-122.

32. Benzagmout M, Gatignol P, Duffau H. Resection of World Health Organization Grade II gliomas involving Broca's Area: Methodological and Functional Considerations. Neurosurgery. 2007; 61 (4): 741-742

33. Lubrano V, Draper L, Roux FE. What Makes Surgical Tumor Resection Feasible in Broca's Area? Insights into Intraoperative Brain Mappin. Neurosurgery. 2010; 66 (5): 868-875.

34. Rolston JD, Englot DJ, Benet A, Li J, Cha S, Berger MS. Frontal Operculum Gliomas: Language Outcome Following Resection. J Neurosurg. 2015; 122 (4): 725-734. 
35. Duffau H, Capelle L, Denvil D, Peggy G, Sichez N, Van Effenterre R, The Role of Dominant Premotor Cortex in Language: A Study Using Intraoperative Functional Mapping in Awake Patients.

Neuroimage. 2003; 20 (4): 1903-1914.

36. Gajardo-Vidal A, Lorga-Puls DL, PLORAS team, Warner H, Pshdary B,Crinion JT, et al. Damage to Broca's Area does not contribute to long-term speech production outcome after stroke. Brain. 2021. 116.

37. Kim JH, Amankulor NM, Peck KK, Brennan N, Gutin PH, Holodny Al. Resection of glioma in an fMRIdefined "split" Broca's area. Neurocase. 2014;20(5):481-486.

38. Sanai N, Mirzadeh Z, Berger MS. Functional Outcome after Language Mapping for glioma Resection. N Engl J Med. 2008; 358: 18-27.

39. Fiebach CJ, Schubotz RI. Dynamic Anticipatory Processing od Hierarchical Sequential Events: A Common Role for Broca's Area and Ventral Premotor Cortex Across Domains?. Cortex, 2006; 42 (4): 499-502.

40. Van Geemen K, Herbet G, Moritz-Gasser S, Duffau H. Limited plastic potential of the left ventral premotor cortex in speech articulation: evidence from intraoperative awake mapping in glioma patients. Hum Brain Mapp. 2014;35(4):1587-1596.

\section{Tables}

Due to technical limitations, tables are only available as a download in the Supplemental Files section.

\section{Figures}



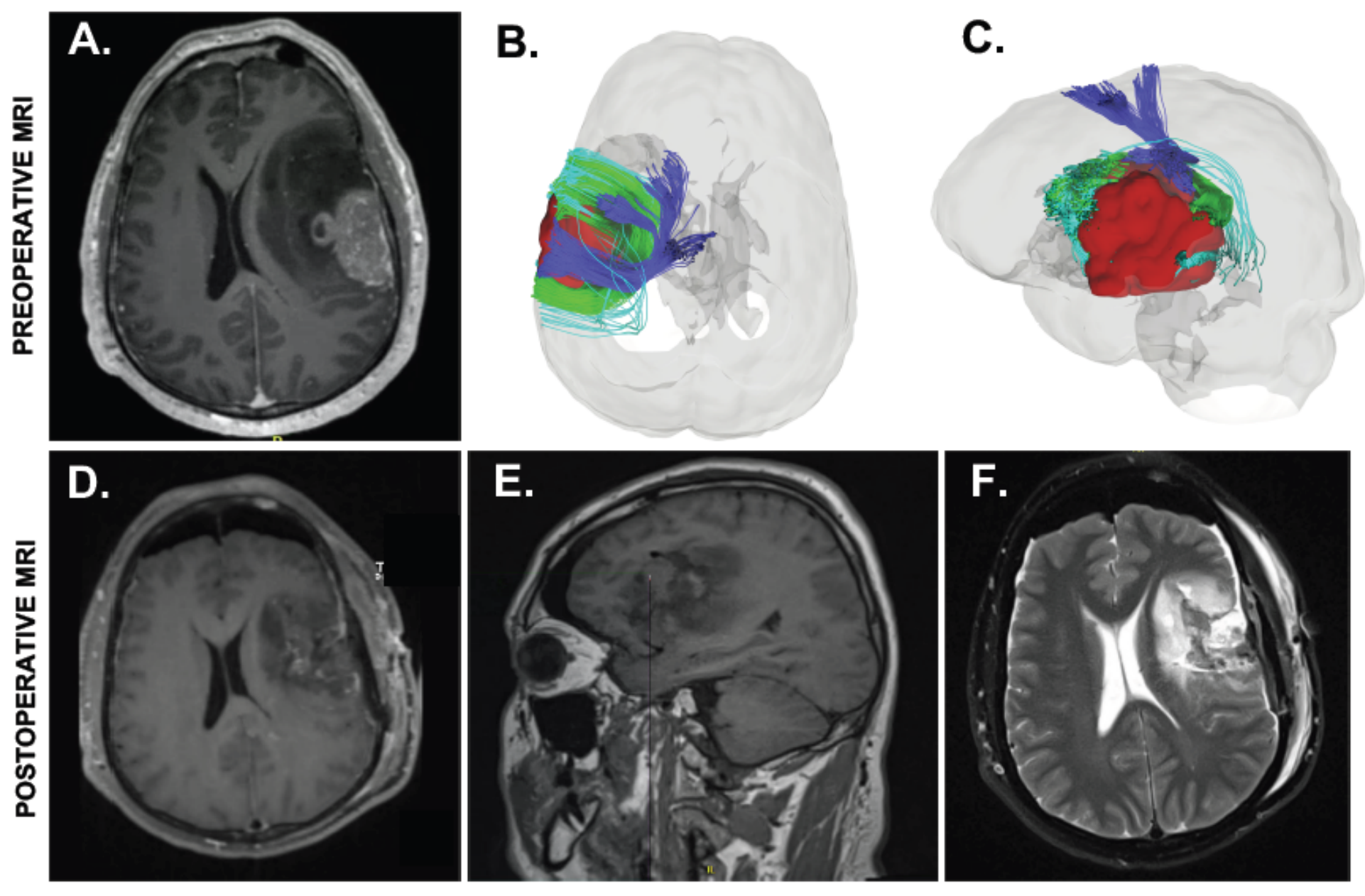

\section{Figure 1}

Representative case.40-year-old, left-handed patient with a 5-year history of intermittent speech arrest, reduced verbal working memory and episodes of postictal aphasia, that became more evident 6 months before diagnosis. Preoperative imaging (A) showed a large enhancing mass lesion in the left IFG with extensive surrounding vasogenic edema and mass effect with persistent left-to-right midline shift measuring approximately $9 \mathrm{~mm}$. Diffusion tractography $(B, C)$ shows superior and posterior displacement of the frontal aslant tract (FAT; blue) with splaying of the anterior and posterior portions of the arcuate fasciculus (AF; cyan) and superior longitudinal fasciculus 3 (SLF3; green) around the tumor (red). Both AF and SLF3 surround the anterior, deep, and posterior margin of the tumor. Patient was taken to the OR for a left frontoparietal awake craniotomy with intraoperative mapping for tumor resection. During surgery, he presented transient semantic paraphasicerrors near the SLF3, AF, and the insula. At the end of the surgery, he remained at his baseline neuropsychological evaluation, and no new surgical-related deficits were noted after the procedure. Postoperative imaging (D-F) showed gross total resection of the lesion (EOR 97.86\%) with minimal residual in the insula and the vPMC, mild edema along the medial aspect of the postoperative cavity and expected FLAIR residual abnormalities. Diagnosis was an IDH mutant (WHO grade IV) glioblastoma. At 2-week follow up, the patient remained neurologically intact and underwent the Stupp protocol for treatment. 

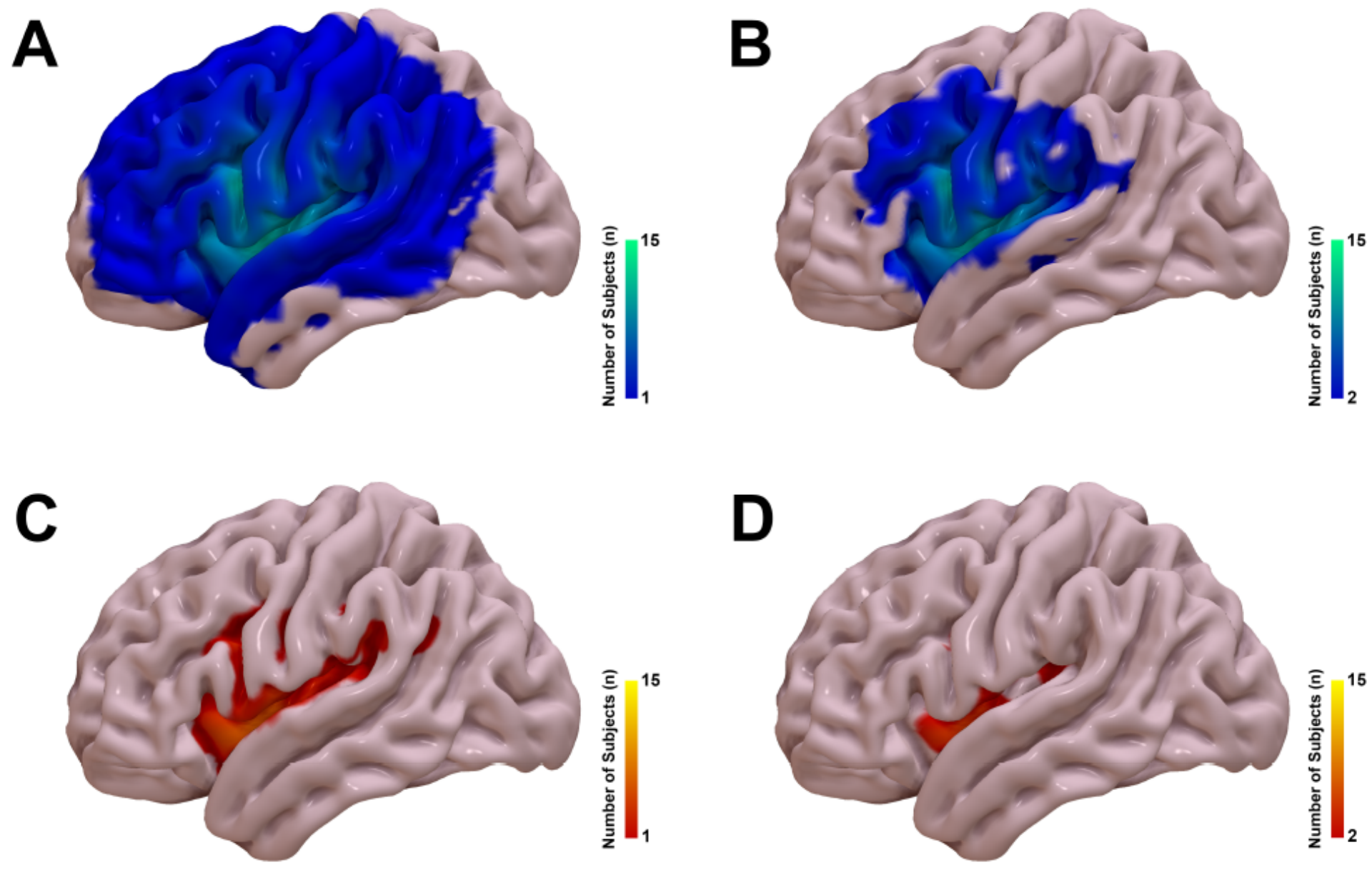

Figure 2

.(A) Preoperative probability map of combined enhancing and non-enhancing tumor for all patients. (B) Preoperative probability map of combined enhancing and non-enhancing tumor thresholded at $93 \%$ ( $n>$ 1). (C) Postoperative probability map of residual enhancing and non-enhancing tumor thresholded at the 93rd percentile $(n>1)$ and the (D) 87th percentile $(n>2)$ show complete resection of tumor within the IFG in the majority of cases. Residual tumor was greatest in the insular and subinsular regions. 

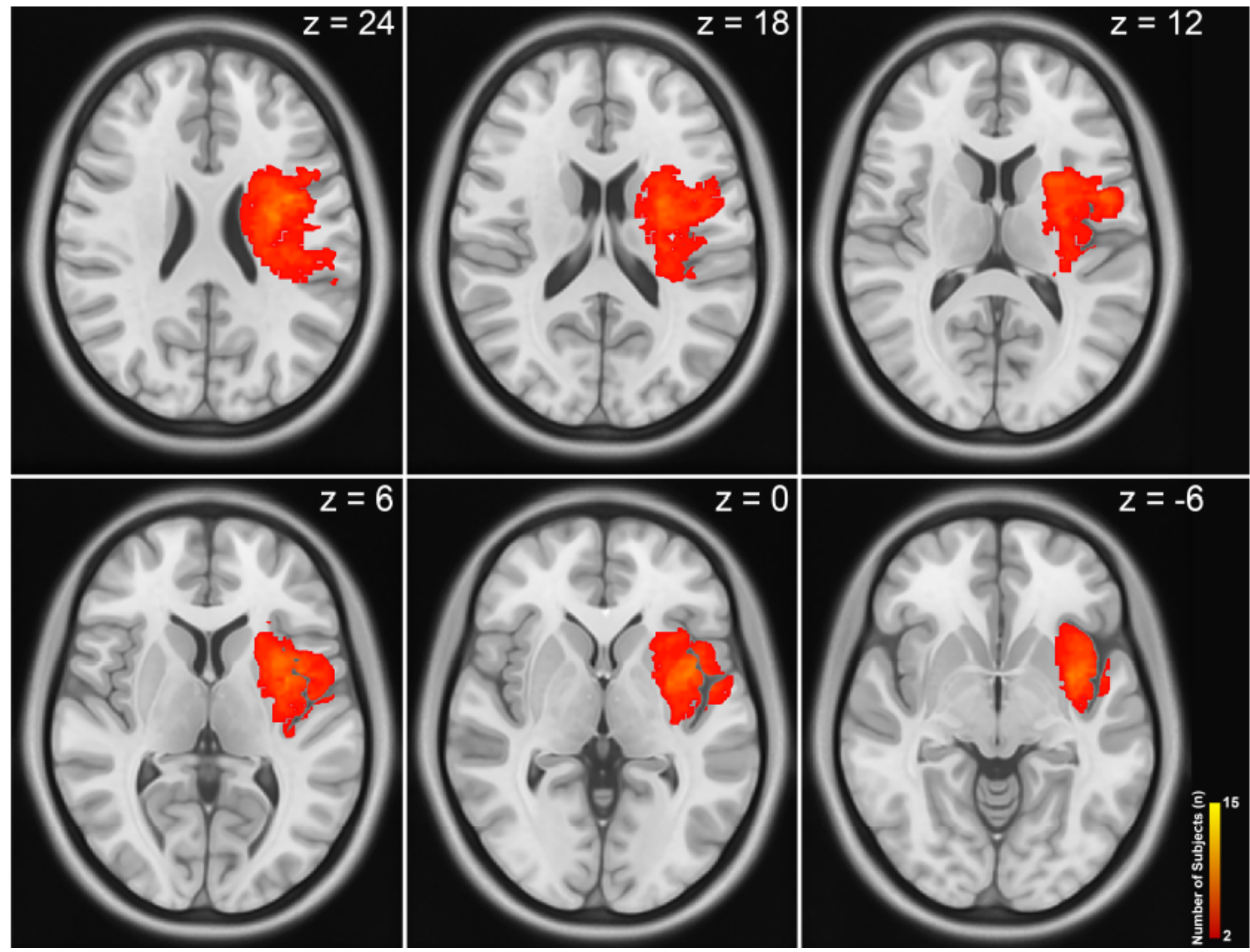

Figure 3

Axial images at specified z-axis coordinates in Montreal Neurological Institute (MNI) template space showing the postoperative probability map of residual enhancing and non-enhancing tumor thresholded at the 87th percentile $(n>2)$. Residual tumor was greatest in the insular and subinsular regions, as well as the deep margins within critical white matter regions of the centrum semiovale. 

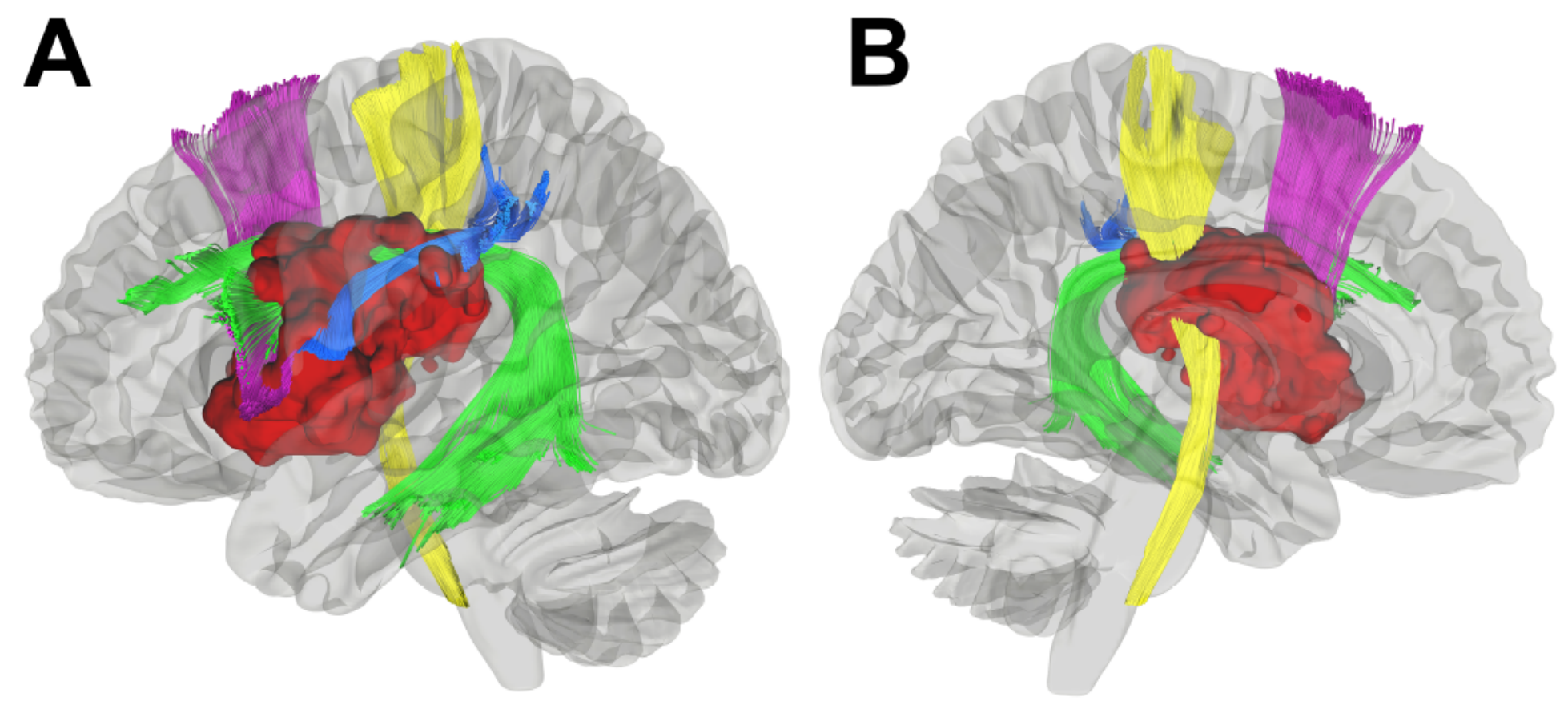

Figure 4

Relationship of the residual enhancing and non-enhancing tumor (red; thresholded at the 87th percentile $(n>2))$ to critical left hemispheric white matter tracts. Purple $=$ frontal aslant tract; yellow $=$ corticospinal tract; blue = superior longitudinal fasciculus III; green = arcuate fasciculus

\section{Supplementary Files}

This is a list of supplementary files associated with this preprint. Click to download.

- Table1IFGPSM.pdf

- Table2IFGPSM.pdf

- Table3IFGPSM.pdf

- Table4IFGPSM.pdf 